\title{
Bovine Model of Doxorubicin-Induced Cardiomyopathy
}

\author{
Carlo R. Bartoli, ${ }^{1,2,3}$ Kenneth R. Brittian, ${ }^{3}$ Guruprasad A. Giridharan, ${ }^{4}$ Steven C. Koenig, ${ }^{2,4}$ \\ Tariq Hamid, ${ }^{3}$ and Sumanth D. Prabhu ${ }^{3,5,6}$
}

${ }^{1}$ MD/PhD Program, Department of Physiology and Biophysics, University of Louisville, Louisville, KY 40202, USA

${ }^{2}$ Cardiovascular Innovation Institute, University of Louisville, Louisville, KY 40202, USA

${ }^{3}$ Department of Medicine, Institute of Molecular Cardiology, University of Louisville, Louisville, KY 40202, USA

${ }^{4}$ Department of Bioengineering, University of Louisville, Louisville, KY 40208, USA

${ }^{5}$ Department of Physiology and Biophysics, University of Louisville, Louisville, KY 40202, USA

${ }^{6}$ Robley Rex VAMC, Louisville, KY 40206, USA

Correspondence should be addressed to Sumanth D. Prabhu, sprabhu@louisville.edu

Received 25 August 2010; Revised 29 October 2010; Accepted 16 November 2010

Academic Editor: Andrea Vecchione

Copyright () 2011 Carlo R. Bartoli et al. This is an open access article distributed under the Creative Commons Attribution License, which permits unrestricted use, distribution, and reproduction in any medium, provided the original work is properly cited.

Left ventricular assist devices (LVADs) constitute a recent advance in heart failure (HF) therapeutics. As the rigorous experimental assessment of LVADs in HF requires large animal models, our objective was to develop a bovine model of cardiomyopathy. Male calves $(n=8)$ were used. Four animals received $1.2 \mathrm{mg} / \mathrm{kg}$ intravenous doxorubicin weekly for seven weeks and four separate animals were studied as controls. Doxorubicin-treated animals were followed with weekly echocardiography. Target LV dysfunction was defined as an ejection fraction $\leq 35 \%$. Sixty days after initiating doxorubicin, a terminal study was performed to determine hemodynamic, histological, biochemical, and molecular parameters. All four doxorubicin-treated animals exhibited significant $(P<0.05)$ contractile dysfunction, with target LV dysfunction achieved in three animals. Doxorubicin-treated hearts exhibited significantly reduced coronary blood flow and interstitial fibrosis and significantly increased apoptosis and myocyte size. Gene expression of atrial natriuretic factor increased more than 3-fold. Plasma norepinephrine and epinephrine levels were significantly increased early and late during the development of cardiomyopathy, respectively. We conclude that sequential administration of intravenous doxorubicin in calves induces a cardiomyopathy with many phenotypic hallmarks of the failing human heart. This clinically-relevant model may be useful for testing pathophysiologic responses to LVADs in the context of HF.

\section{Introduction}

Many insights into the biological mechanisms of left ventricular (LV) remodeling and heart failure (HF) have been derived from small animals, particularly rodents such as mice. However, establishing direct analogies between rodents and humans can be problematic as there are considerable differences in cardiac physiology (e.g., heart rate, oxygen consumption, regulatory proteins, contractile proteins, stem cell characteristics, etc.) between species [1]. Validation and proper translation of fundamental discoveries into clinical utility necessitates the use of large animal models that more closely approximate human physiology $[1,2]$.
A recent advance in HF therapeutics consists of mechanical unloading with LV assist devices (LVADs). A large animal disease model that mimics the human cardiovascular condition (bovine or ovine) [3] is particularly critical for the rigorous evaluation of (patho)physiological responses induced by LVADs, as phylogenetically lower species cannot physically accommodate implantable cardiac devices. Moreover, prolonged mechanical unloading with LVADs can reverse remodeling of the human failing LV [4], with reports of myocardial recovery sufficient for LVAD explantation in a subset of patients $[5,6]$. Meaningful assessment of the hemodynamic and molecular mechanisms of LVAD-induced myocardial recovery and the influence of different strategies 
of mechanical unloading requires the use of a reliable large animal model of HF.

In view of both anatomical size of the thoracic cavity and cardiovascular physiology, the current industry standard is to use normal calves to test safety, performance, and reliability of LVAD systems. However, evaluation of these devices in animals with chronic HF, with attendant differences in cardiac physiology and hemodynamics from the normal condition, has been extremely limited [7-9]. As myocardial recovery most often has been reported in patients with idiopathic dilated cardiomyopathy [5], our objective in this study was to develop a nonischemic bovine model of cardiomyopathy to provide a substrate for studying device-based therapies for HF. For this purpose, we used doxorubicin, a broad-spectrum antineoplastic drug with dose-dependent cardiotoxicity that has been used to induce chronic HF in several animal species that include sheep $[10,11]$ and dogs [12-17].

\section{Methods}

2.1. Animals. All animals received humane care and were handled in accordance with National Institutes of Health and University of Louisville Animal Care Committee guidelines. Experimental procedures followed animal study protocols approved by the University of Louisville Institutional Animal Care and Usage Committee.

Male Jersey and mixed-breed calves ( $n=8,4-6$ months old, $88 \pm 6 \mathrm{~kg}$ ) were used. Four animals were administered intravenous doxorubicin $1.2 \mathrm{mg} / \mathrm{kg}$ once weekly via the external jugular vein for seven weeks. One animal that was relatively refractory to doxorubicin treatment received a dose of $1.5 \mathrm{mg} / \mathrm{kg}$ for the last two doses. For 48 hours following each injection, animals were given $100 \mathrm{mg}$ oral atenolol daily to prevent arrhythmia [19]. Four animals were used as controls for myocardial blood flow, histological, and molecular analyses. A separate group of 9 normal, agematched calves from a previous study in our laboratory [18] were used for hemodynamic comparison with the doxorubicin group. Serial venous blood samples were drawn from doxorubicin-treated animals at baseline, 14-day-, and 45-day-time points during the doxorubicin administration protocol.

2.2. Echocardiography. Transthoracic M-mode, 2D, and Doppler echocardiograms were measured at baseline and weekly during the doxorubicin protocol with a Phillips iE33 machine with S8-3 ultrasound probe. Prior to the final study and termination, open-chest epicardial echocardiography was performed. Modified parasternal and apical fourchamber views were used for imaging, and LV ejection fraction (EF) was determined using the modified Simpson technique (summation of discs). Target LV dysfunction was defined as an LVEF of less than or equal to $35 \%$.

2.3. Surgical Preparation for Hemodynamic Measurement. Within 10 days of the final doxorubicin injection, a terminal study was performed to measure cardiovascular hemodynamics and to harvest tissues for myocardial blood flow, histology, and molecular analysis. Animals were preanesthetized with intravenous atropine $(30 \mathrm{mg})$, anesthetized with inhaled isoflurane (3-5\%), and anticoagulated with intravenous heparin (100 units/kg). Fluid-filled arterial and venous catheters were placed in the right carotid artery and jugular vein for blood sampling. A left thoracotomy was performed and ribs no. 4 and no. 5 were resected. High-fidelity micromanometer catheters (Millar Instruments) were placed in the left atrium (single-tip) and across the aortic valve (dual-tip) for simultaneous measurement of left atrial (LA), $\mathrm{LV}$, and aortic blood pressure. Transit-time ultrasonic flow probes (Transonics) were placed around the pulmonary artery and left main coronary artery to measure cardiac output and volumetric coronary blood flow, respectively.

2.4. Microsphere Protocol. A silicone catheter (7 Fr; Access Technologies) was advanced $4 \mathrm{~cm}$ into the LA appendage chamber. This depth and the angle of catheter entry parallel to the surface of the atrial appendage ensured that the catheter did not interfere with mitral valve function. An aortic catheter oriented downstream was placed in the aorta as previously described [20]. The LA catheter was used for injection of multiple colors of fluorescent-labeled $15 \mu \mathrm{m}$ microspheres into the systemic arterial circulation. Simultaneously, a reference blood sample was withdrawn at a known rate $(15 \mathrm{~mL} / \mathrm{min})$ from the aorta as previously described [20].

The microsphere technique enables the precise measurement of regional blood flow in vascular beds of interest. Microspheres injected into the LA chamber mix with blood in the LV and are subsequently ejected into the aorta, from where they disseminate throughout the body and lodge within the smallest precapillary arterioles based on regional tissue blood flow distribution. We have previously demonstrated that with the quantity used, $15 \mu \mathrm{m}$ spheres do not cause ischemia and do not induce pathology [20]. The aortic blood sample acts as a reference for later determination of flow in tissues of interest. The number of counted microspheres in the reference blood sample (known) is compared to the number of microspheres that lodge and are counted in a tissue sample of interest (known). The ratio between the two sphere counts is equal to the ratio between the calibrated rate of aortic withdrawal (known) and flow in the tissue of interest (unknown) and provides accurate tissue-specific blood flow in $\mathrm{mL} / \mathrm{min} / \mathrm{g}[20,21]$.

2.5. Quantification of Microspheres. At the completion of the study, while under anesthesia, euthanasia was performed with a single fatal bolus injection of BeuthanasiaD Special $(1 \mathrm{~mL} / 5 \mathrm{~kg} \mathrm{IV})$. The heart was removed and weighed. One- to two-gram tissue sections from the LV-free wall, right ventricular(RV)-free wall, and interventricular septum together with reference blood samples were sent to IMT/Stason Laboratories (Irvine, CA) for automated digestion and counting of fluorescent microspheres with flow cytometry and calculation of tissue-specific blood flows. 
TABLE 1: Primers used for real-time PCR.

\begin{tabular}{lcc}
\hline Gene & Forward Primer & Reverse Primer \\
\hline ANF & $5^{\prime}$-CAGGGCAAACAGGAGCAAA-3' & $5^{\prime}$-TCCATCAGGTCTGCATTGGA-3' \\
CTGF & $5^{\prime}$-TCCCACGGAGGGTCAAACT-3' & $5^{\prime}$-CATCACGGGACACCCATTC-3' \\
MMP-2 & $5^{\prime}$-CCTGGGCCCCGTCACT-3' & $5^{\prime}$-GAGATGCCGTCGAAGACGAT-3' \\
MMP-9 & $5^{\prime}$-TTAGGAACCGCTTGCATTTCTT-3' & $5^{\prime}$-CCCCCTCCCTCAGAAAGTCT-3' \\
18s rRNA & $5^{\prime}$-CGAACGTCTGCCCTATCAACTT-3' & $5^{\prime}$-ACCCGTGGTCACCATGGTA-3' \\
\hline
\end{tabular}

ANF, atrial natriuretic factor (NM_174124); CTGF, connective tissue growth factor (NM_174030); MMP-2, matrix metalloproteinase-2 (NM_174745); MMP9, matrix metalloproteinase-9 (NM_174744); 18s rRNA, 18s ribosomal RNA (eukaryotic sequence).

2.6. Hemodynamic Instrumentation and Data Reduction. All pressure and flow transducers were pre- and postcalibrated against known physical standards to ensure measurement accuracy. Data were collected at $400 \mathrm{~Hz}$, signal conditioned, and A/D converted for digital analysis using our GLP compliant data acquisition system [22]. Pressure and flow recordings were used to derive heart rate, cardiac output, mean arterial pressure, mean LA pressure, LV peak systolic and end-diastolic pressure (EDP), peak $\pm \mathrm{dP} / \mathrm{dt}, \mathrm{LV}$ external work, and mean diastolic coronary artery blood flow. These parameters were calculated on a beat-to-beat basis for each 30-second data set with the Hemodynamic Evaluation and Assessment Research Tool (HEART) program developed in Matlab (Version 6.5, MathWorks). All analyzed beats in each data set (approximately $30-50$ beats) were averaged to obtain a single representative mean value for each calculated parameter.

2.7. Histological Assessment. Paraffin-embedded tissue sections $(4 \mu \mathrm{m})$ from the LV, RV, and interventricular septum were deparaffinized, rehydrated, and stained with Masson's Trichrome (for collagen) with standard histological techniques as previously described [23]. To determine myocyte cross-sectional area, FITC-conjugated wheat germ agglutinin (Molecular Probes) staining of cell membranes together with DAPI (Molecular Probes) nuclear costaining was performed as previously described [24]. Myocyte area determined from an average of $\sim 100-150$ cross-sectional cells with centrally located round nuclei and the total fibrotic area were assessed using Metamorph Imaging Software.

Apoptosis in cardiac tissue was determined with the DeadEnd Fluorometric TUNEL System (Promega), which catalytically incorporates fluorescein-12-dUTP at DNA strand breaks as previously described [24]. All sections were counterstained with DAPI (Molecular Probes) at a final concentration of $2 \mu \mathrm{M}$. Images were viewed with epifluorescence microscopy (Nikon TE2000) within 24 hours and analyzed with Metamorph Imaging Software.

2.8. Myocardial Gene Expression. mRNA expression in the heart was quantified by real-time polymerase chain reaction (PCR) as previously described [23, 25]. Briefly, total RNA was isolated from LV tissue with TRIzol reagent (Invitrogen), and cDNA was synthesized from $1 \mu \mathrm{g}$ RNA with the iScript cDNA Synthesis kit (BioRad). Relative levels of mRNA transcripts for atrial natriuretic factor (ANF), connective tissue growth factor (CTGF), matrix metalloproteinase (MMP)-2, and MMP-9 were quantified by real-time PCR with the use of SYBR Green (Applied Biosystems) and the sense/antisense primer pairs listed in Table 1. Data were normalized to $18 \mathrm{~s}$ ribosomal RNA subunit expression using the $\Delta \Delta \mathrm{C}_{\mathrm{T}}$ comparative method, and the values from doxorubicin-treated hearts were expressed as a fold change over control.

2.9. Measurement of Plasma Catecholamines. Plasma norepinephrine and epinephrine levels were determined by colorimetric quantitative competitive ELISA with a commercially available kit (Rocky Mountain Diagnostics) according to the manufacturer's instructions. Briefly, the derivatized standards, test samples, and the solid phase bound analytes competed for a fixed number of antiserum binding sites. After washing of the free antigen and the antigen-antiserum complexes, the antibody bound to the solid phase was detected by a peroxidase-conjugated secondary antibody. Quantification of unknown samples was then extrapolated from a reference standard curve.

2.10. Statistics. Serial echocardiographic and catecholamine data from the same animal at different time points during the doxorubicin protocol were compared using one-way ANOVA with Tukey posttest. Hemodynamic, myocardial blood flow, histological, and molecular comparisons between doxorubicin-treated animals and normal animals were performed with an unpaired $t$-test. A $P$-value $<0.05$ was considered statistically significant. All continuous data are reported as mean \pm standard deviation.

\section{Results}

3.1. Clinical Findings. All four animals developed chronic coughing, three of four animals developed dyspnea on exertion, and one animal developed dyspnea at rest and severe ascites. During the duration of the study, three of four animals lost weight and exhibited signs of chronic dehydration.

3.2. Echocardiography. The target LVEF $(\leq 35 \%)$ was achieved in three animals, and diminished heart function was induced in the remaining animal treated with doxorubicin. 


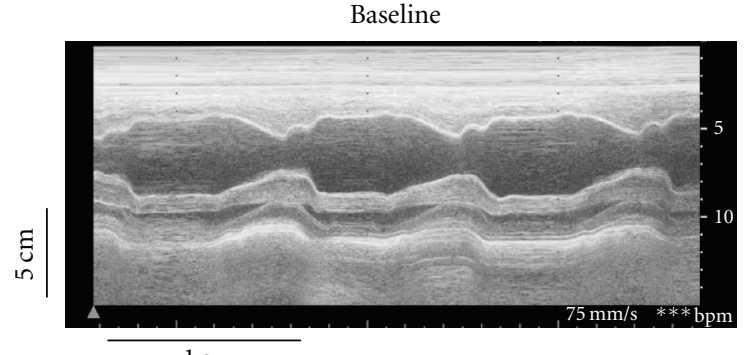

$1 \mathrm{~s}$

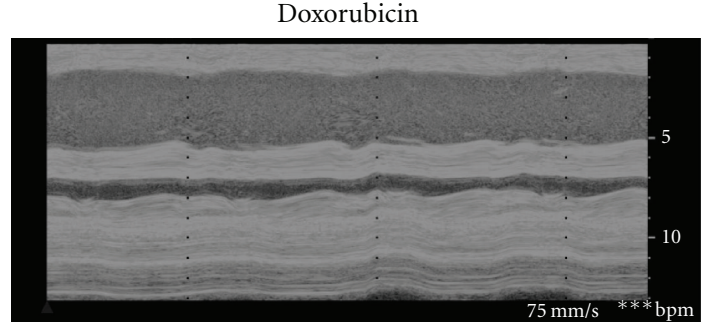

(b)

Figure 1: M-mode echocardiographic images from the same animal at baseline (a) and 60 days after initiation of weekly intravenous doxorubicin (b).

TABLE 2: Serial echocardiography in doxorubicin-treated calves.

\begin{tabular}{lccc}
\hline & Baseline & Final awake & Final anesthetized \\
\hline $\operatorname{LVEDV}(\mathrm{mL})$ & $58 \pm 2$ & $73 \pm 22$ & $75 \pm 25$ \\
$\operatorname{LVESV}(\mathrm{mL})$ & $5 \pm 4$ & $24 \pm 14^{*}$ & $46 \pm 17^{* \#}$ \\
$\operatorname{LVEF}(\%)$ & $91 \pm 6$ & $64 \pm 23^{*}$ & $36 \pm 23^{* \#}$ \\
\hline
\end{tabular}

$\mathrm{LV}$, left ventricular; EDV, end-diastolic volume; ESV, end-systolic volume; EF, ejection fraction. ${ }^{*} P<0.05$ versus baseline; ${ }^{\#} P<0.05$ versus final awake. $n=4$.

TABle 3: Hemodynamics in doxorubicin-treated and normal calves.

\begin{tabular}{lcc}
\hline & Normal $^{\ddagger}$ & Doxorubicin treated \\
\hline CO $(\mathrm{L} / \mathrm{min})$ & $8.1 \pm 1.2$ & $5.8 \pm 2.4^{*}$ \\
MAP $(\mathrm{mm} \mathrm{Hg})$ & $90 \pm 10$ & $70 \pm 17^{*}$ \\
LAP $(\mathrm{mm} \mathrm{Hg})$ & $14 \pm 6$ & $15 \pm 9$ \\
LVPSP $(\mathrm{mm} \mathrm{Hg})$ & $103 \pm 9$ & $88 \pm 22$ \\
LVEDP $(\mathrm{mm} \mathrm{Hg})$ & $16 \pm 6$ & $15 \pm 12$ \\
peak $+\mathrm{dP} / \mathrm{dt}(\mathrm{mm} \mathrm{Hg} / \mathrm{s})$ & $1252 \pm 407$ & $767 \pm 222^{*}$ \\
peak $-\mathrm{dP} / \mathrm{dt}(\mathrm{mm} \mathrm{Hg} / \mathrm{s})$ & $-2528 \pm 866$ & $-1135 \pm 610^{*}$ \\
LVEW $(\mathrm{mm} \mathrm{Hg} \cdot \mathrm{ml})$ & $8758 \pm 2441$ & $6001 \pm 2751$ \\
CAF $(\mathrm{mL} / \mathrm{min})$ & $207 \pm 138$ & $77 \pm 16$ \\
\hline
\end{tabular}

$\mathrm{CO}$, cardiac output; MAP, mean arterial pressure; LAP, left atrial pressure; LV, left ventricular; PSP, peak systolic pressure; EDP, end diastolic pressure; EW, external work; CAF, coronary artery flow.

${ }^{\ddagger}$ normal calves from [18]. ${ }^{*} P<0.05$ versus normal.

Representative M-Mode echocardiographic images from one animal at baseline and 60 days after initiating doxorubicin are shown in Figure 1. Mild LV dilitation was observed along with a much more profound reduction in systolic contraction and shortening fraction. Group data $(n=4)$ are shown in Table 2. Doxorubicin induced mild increases in LV end-diastolic volume (EDV) that were not statistically significant, but significant $(P<0.05)$ increases in LV end-systolic volume (ESV) and worsening of LVEF were observed. These effects were more profound under isoflurane anesthesia. In the subset of animals that achieved target LVEF, there was significant exacerbation of all echocardiographic parameters of interest (baseline versus final anesthetized: LVEDV $57 \pm 2$ versus $71 \pm 29 \mathrm{~mL}$; LVESV $6 \pm 3$ versus $52 \pm$ $15 \mathrm{~mL}$; LVEF $89 \pm 6$ versus $25 \pm 10 \%$; $P<0.05$ ).

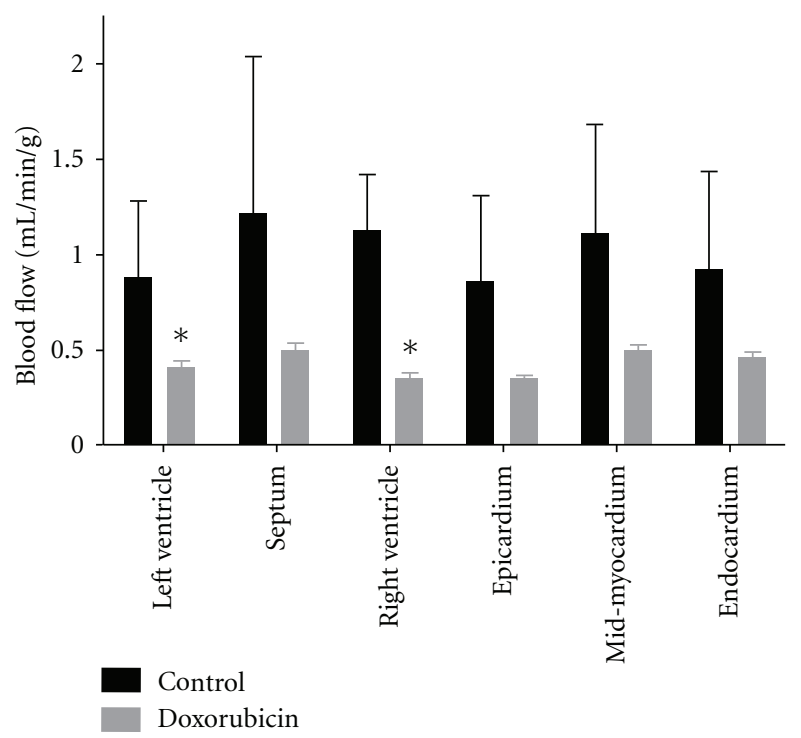

Figure 2: Regional myocardial blood flow was determined with $15 \mu \mathrm{m}$ microspheres in control and doxorubicin-treated animals. ${ }^{*} P<0.05$ versus control; $n=4$ /group.

3.3. Hemodynamics. Table 3 depicts hemodynamic parameters measured 60 days after the initiation of doxorubicin. As compared to normal calves of somewhat smaller size ( $n=9$, mean weight: $76.1 \mathrm{~kg})$ that were previously studied in our laboratory [18], doxorubicin-treated calves exhibited hemodynamic changes indicative of LV contractile dysfunction with significantly $(P<0.05)$ decreased cardiac output, mean arterial pressure, peak $+\mathrm{dP} / \mathrm{dt}$, and peak $-\mathrm{dP} / \mathrm{dt}$, and a trend toward decreased LV peak systolic pressure $(P=$ $0.10)$, LV external work $(P=0.10)$, and left main coronary artery blood flow $(P=0.12)$. Interestingly, despite significant inotropic and lusitropic depression, filling pressures (LA pressure and LV end-diastolic pressure) were comparable between the groups.

3.4. Regional Myocardial Blood Flow. Figure 2 demonstrates regional myocardial blood flow in the LV-free wall, interventricular septum, RV-free wall, and the transmural distribution in control animals and doxorubicin-treated animals 


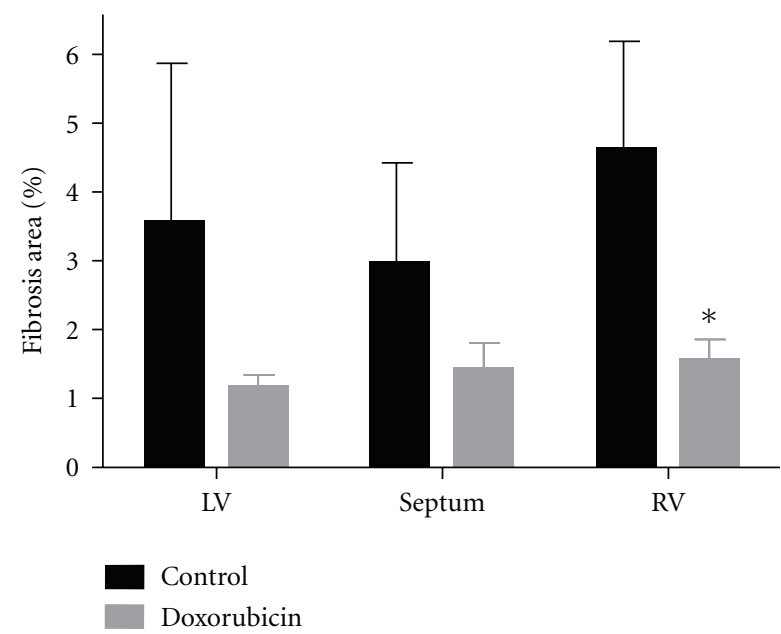

(a)

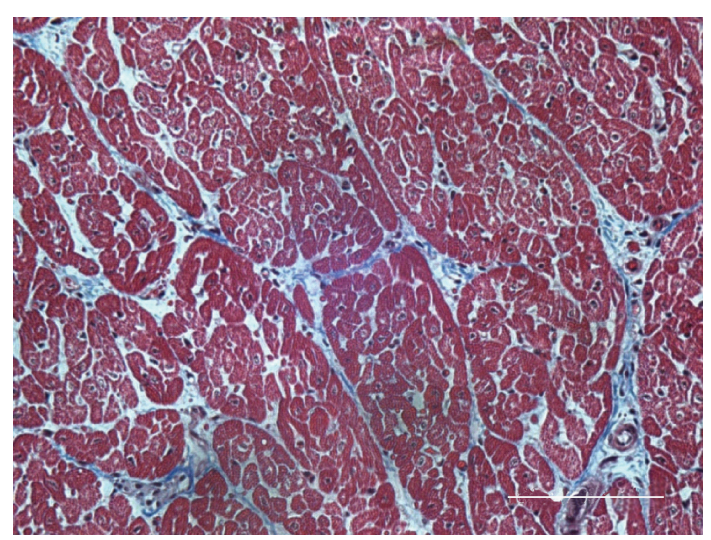

Control, 40x

(b)

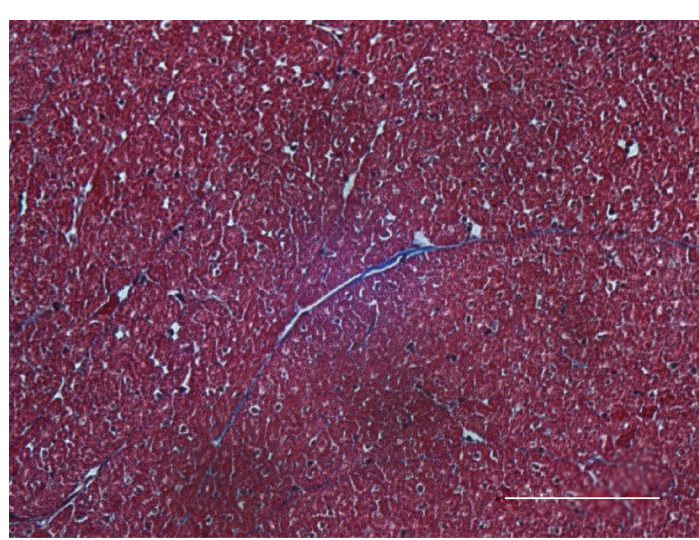

Doxorubicin, $40 \mathrm{x}$

(c)

Figure 3: (a) Interstitial fibrosis as determined by Masson's Trichrome staining in control and doxorubicin-treated hearts. (b) and (c) Representative histomicrographs of myocardial architecture in control and doxorubicin-treated hearts. In control animals, the outlines of individual myocytes are apparent. Interstitial endo- and perimysium are well established and clearly visible. In doxorubicin treated animals, normal myocyte borders are not apparent, and connective tissue is largely absent. $40 \mathrm{x}$, white bar $=50 \mu \mathrm{m},{ }^{*} P<0.05 \mathrm{versus}$ control. LV, left ventricle; RV, right ventricle; $n=4$ /group.

at 60 days after initiation of doxorubicin. There were statistically significant $(P<0.05)$ reductions in myocardial blood flow in the LV- and RV-free walls and trends toward reduced blood flow in the septum $(P=0.13)$, epicardium $(P=0.07)$, midmyocardium $(P=0.07)$, and endocardium $(P=0.13)$.

3.5. Histology and Gene Expression. Gross heart weights were $518 \pm 82 \mathrm{~g}$ for control animals and $604 \pm 90 \mathrm{~g}$ for animals treated with doxorubicin and were not statistically different as a direct comparison $(P=0.25)$ or normalized to body weight $(P=0.88)$ and suggest the absence of doxorubicininduced hypertrophy at the chamber level.

Figure 3 demonstrates the degree of collagen deposition/fibrosis in the LV, RV, and interventricular septum as determined by Masson's Trichrome staining. As compared to control hearts, after doxorubicin treatment, connective tissue decreased or tended to decrease in each of the three regions (LV $P=0.14$, septum $P<0.10$, RV $P<$ $0.01)$. Moreover, as seen in the accompanying representative histomicrographs, the outline of individual myocytes and the interstitial space were well established and clearly visible in control hearts. In contrast, doxorubicin-treated hearts did not exhibit clear myocyte borders, and interstitial connective tissue volume was markedly diminished. To explore this further, we examined myocardial gene expression of CTGF, a profibrotic matrix-associated protein, and MMP-2 and -9, which would be expected to augment collagen degradation (Figure 4) [23]. As compared with control hearts, CTGF expression was unchanged in doxorubicin-treated hearts, whereas MMP-2 expression was significantly $(P<0.05)$ upregulated. MMP-9 expression tended toward a decrease $(P=0.09)$. The large increase in MMP-2 would be expected to favor collagen turnover and loss, which was consistent with the histological findings in Figure 3. 


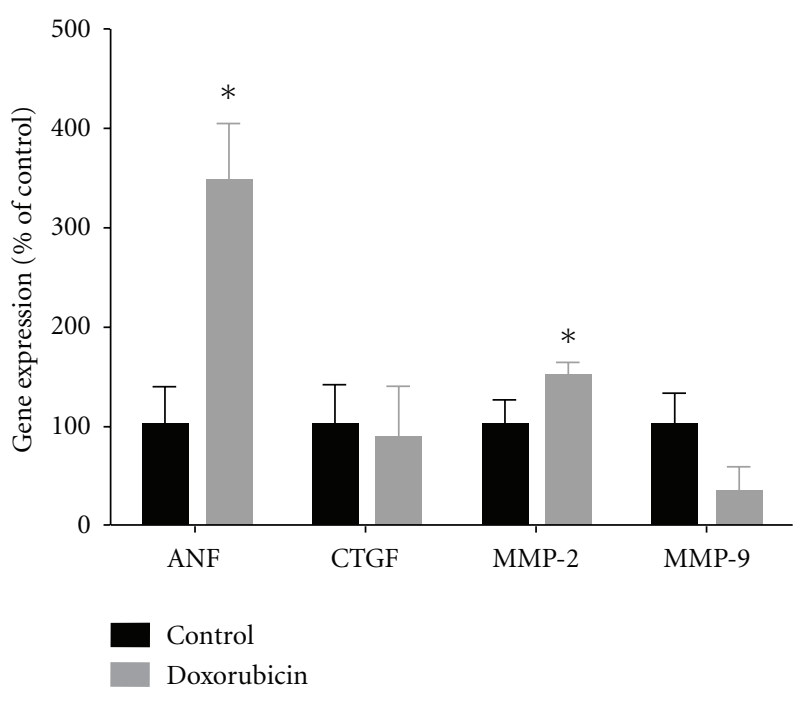

FIGURE 4: mRNA expression for atrial natriuretic factor (ANF), connective tissue growth factor (CTGF), matrix metalloproteinase(MMP-) 2, and MMP-9 in control and doxorubicin-treated hearts as quantified by real-time PCR. Individual gene expression was normalized to $18 \mathrm{~s}$ ribosomal RNA and displayed as a percent of control. ${ }^{*} P<0.05$ versus control; $n=4$ /group.

Figure 5 shows representative wheat-germ agglutinin staining of cell membranes and corresponding myocyte size in the LV, RV, and interventricular septum from control and doxorubicin-treated hearts. As compared with control hearts, after doxorubicin treatment myocyte size increased in each of the three regions and indicated pathologic myocyte hypertrophy. This increase was statistically significant $(P<$ 0.05 ) in the RV and septum but not statistically significant in the $\operatorname{LV}(P=0.29)$. In control hearts, myocytes were of a uniform size and shape, and interstitial space was clearly visible as dark, variegated bands throughout the myocardium. In doxorubicin-treated hearts, myocytes were hypertrophic and nonuniform in shape. Consistent with these histological findings of hypertrophy, mRNA expression of ANF, a hypertrophic marker, was increased $\sim 3.5$-fold in doxorubicin-treated hearts as compared with control (Figure 4).

One potential explanation for doxorubicin-induced myocyte hypertrophy without changes in LV chamber weight would be the concomitant enhancement of myocardial cell loss. Figure 6 demonstrates representative TUNEL stains from control and doxorubicin-treated hearts and the overall apoptotic rate in the LV, interventricular septum, and RV. As compared to controls, doxorubicin treatment enhanced myocardial apoptosis in the heart. This increase was statistically significant in the left ventricle $(P<0.01)$, of borderline significance in the interventricular septum $(P=$ $0.10)$, and not significant in the right ventricle $(P=$ $0.39)$. Staining morphology and the proximity of cells to vascular structures suggested that the apoptotic cells were a mixture of myocytes, endothelial cells, and immune cells.
3.6. Plasma Catecholamines. As shown in Figure 7, plasma norepinephrine levels were significantly increased 14 days into the doxorubicin administration protocol and subsequently returned to baseline at 45 days. Plasma epinephrine increased progressively during doxorubicin administration and was significantly increased over baseline at 45 days. Collectively, these data indicate heightened circulating catecholamines and adrenergic activation, a hallmark of cardiomyopathy and heart failure [26].

\section{Discussion}

In this study, we report for the first time a stable model of doxorubicin-induced cardiomyopathy in the calf. Specifically, weekly intravenous injections of doxorubicin induced (1) mild LV dilatation with impaired LV contraction and relaxation, (2) reductions in cardiac output without changes in LV filling pressure, (3) reductions in myocardial blood flow, (4) myocyte hypertrophy, (5) cardiac cell apoptosis, (6) loss of interstitial collagen, and (7) an increase in plasma catecholamines. Most of these phenotypic features are hallmarks of the failing human heart. Given that the calf is generally used for preclinical testing of mechanical circulatory support devices, this bovine model may be useful as a platform for testing acute and chronic pathophysiologic responses to LVADs in the context of HF and for the development of adjunct therapies for myocardial recovery.

Doxorubicin is a potent broad-spectrum antineoplastic drug with dose-dependent cardiotoxicity that clinically can manifest as cardiomyopathy and HF [27]. Multiple pathological mechanisms have been proposed for doxorubicininduced cardiomyopathy that include the generation of free radicals, oxidative stress-induced lipid peroxidation and mitochondrial damage, suppression of cardiac gene expression and protein synthesis, augmented release of catecholamines, and cardiomyocyte and endothelial cell apoptosis [27-29]. The large number of mitochondria in the heart and the strong affinity of anthracyclines for the inner mitochondrial membrane phospholipid cardiolipin contribute to the mitochondrial accumulation of doxorubicin and predispose cardiac myocytes to doxorubicin toxicity [30]. As such, multiple investigators have used anthracyclines such as doxorubicin to induce cardiomyopathy and HF in a variety of large animal models that include dogs and sheep [10-17, 31]. Large animal studies that have used sequential weekly doxorubicin doses for a circumscribed period (as was done in our study) report a cardiomyopathy that is progressive over the long term without evidence of spontaneous improvement $[10-13,16]$.

In dogs and sheep, serial doxorubicin administration decreased cardiac output by $15-32 \%[11,12,15,17]$. Similarly, in our bovine model, cardiac output was $28 \%$ lower than normal animals. Moreover, significant contractile and lusitropic dysfunction was evident with $\sim 40 \%$ reduction in peak $+\mathrm{dP} / \mathrm{dt}$ and $\sim 55 \%$ reduction in peak $-\mathrm{dP} / \mathrm{dt}$. Interestingly, LV dilatation, a hallmark of anthracycline cardiotoxicity in rodents [27], was relatively modest in our calf model, and filling pressures were normal despite 


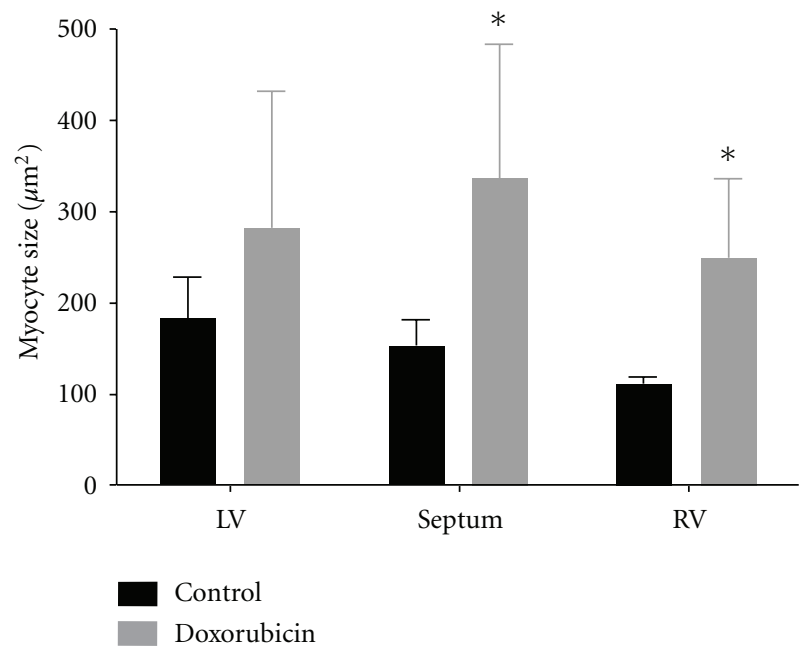

(a)

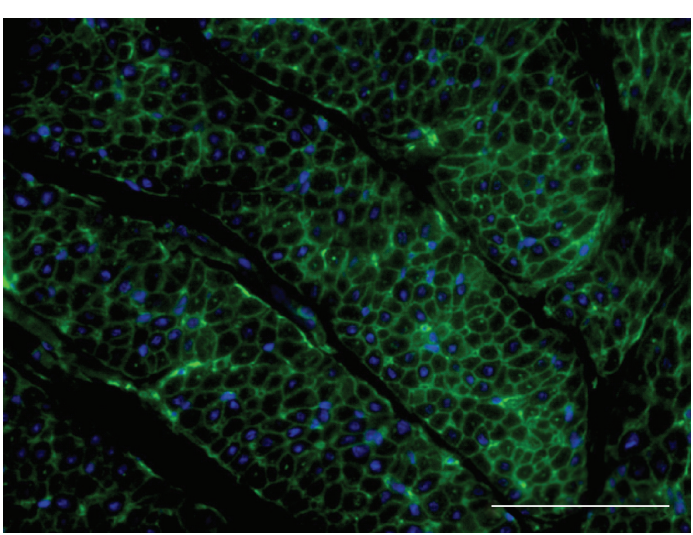

Control, 20x

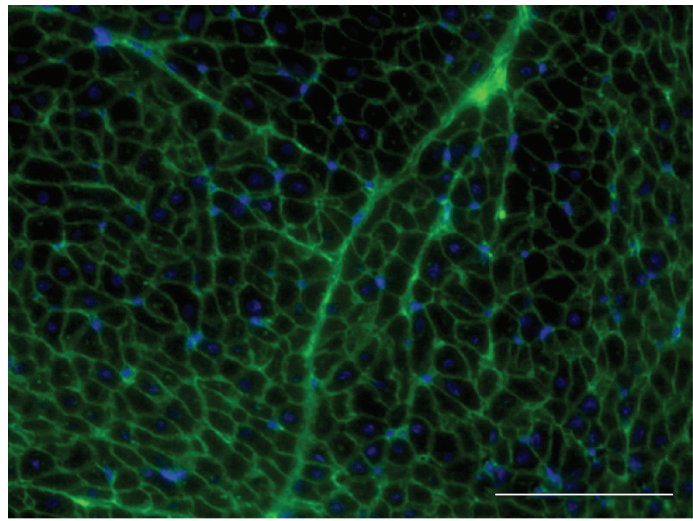

Doxorubicin, 20x

(b)

Figure 5: (a) Myocyte area as determined by wheat germ agglutinin and DAPI costaining in control and doxorubicin-treated hearts. (b) and (c) Representative wheat germ agglutinin and DAPI costaining in control and doxorubicin-treated hearts delineating myocyte cell membranes (green) and nuclei (blue). In control animals, myocytes are of a uniform size and shape, and interstitial space is clearly visible as dark, variegated bands through the myocardium. In doxorubicin-treated animals, myocytes are hypertrophic and nonuniform in shape. 20x, white bar $=100 \mu \mathrm{m},{ }^{*} P<0.05$ versus control. LV, left ventricle; RV, right ventricle; $n=4$ /group.

reduced cardiac output. This suggests that pathological remodeling and hemodynamic decompensation may have become more pronounced upon larger doses of doxorubicin and/or a greater duration of followup. Alternatively, these less pronounced phenotypic features may be species-specific and distinguish doxorubicin-mediated responses in the calf in comparison with other animal species.

LVEF dropped by $\sim 30 \%$ in the conscious state and $\sim 60 \%$ in the anesthetized state as compared with baseline. Importantly, high-baseline LV ejection fraction is normal in calves. The juvenile calf has an accelerated calcium turnover rate and high myocardial contractility [32]. Interestingly, it is well documented that bovine hemodynamics differ between conscious and anesthetized conditions [33, 34]. Normal ejection fraction in calves has been reported to be as high as $85 \pm 9 \%$ in the conscious state [35] and $63 \pm 10 \%$ under anesthetized conditions [33]. In our study, after seven weeks of doxorubicin, the calves exhibited an ejection fraction of $64 \pm 23 \%$ in the conscious state and $36 \pm 23 \%$ under isoflurane anesthesia. LVEF in the conscious state may have overestimated basal mechanical function in these animals, as they were uniformly anxious with attendant increase in stress-induced, catecholamine-mediated effects on cardiac performance. As a result, echocardiographic measurements were performed in a hyperdynamic state as occurs during a stress echocardiogram. Both echocardiographic and hemodynamic measurement in the anesthetized state confirmed significant systolic dysfunction after doxorubicin administration. Moreover, doxorubicin-treated animals exhibited several characteristic histological, biochemical, and molecular features of pathological cardiac remodeling and myocyte hypertrophy. 


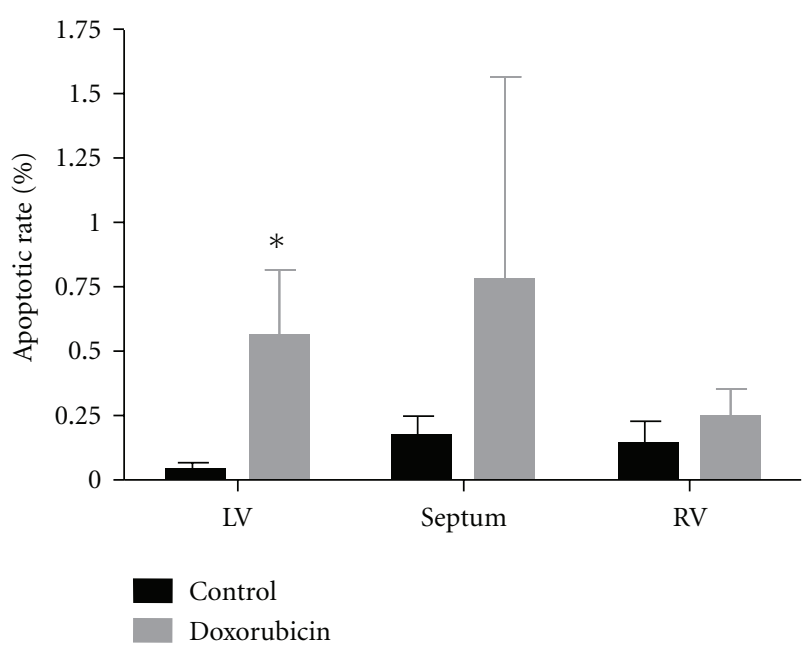

(a)

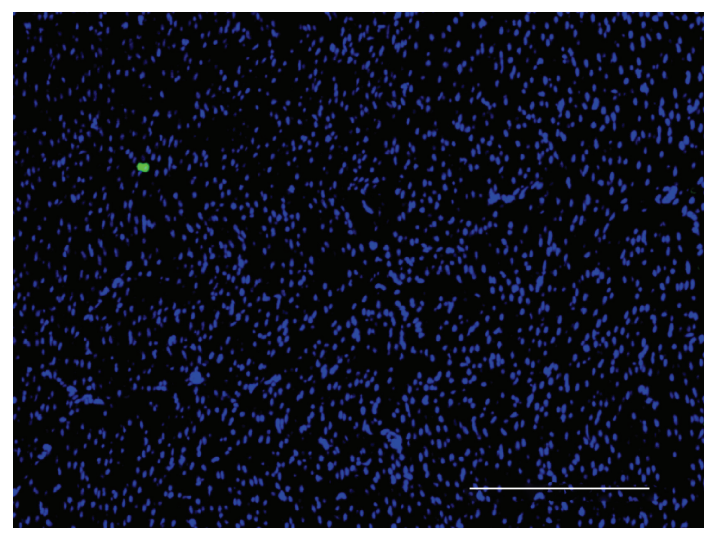

Control, 10x

(b)

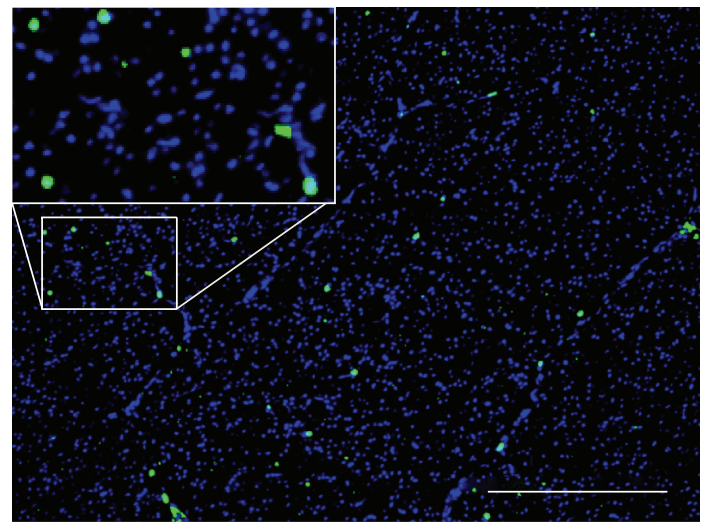

Doxorubicin, $10 \mathrm{x}$

(c)

FIgURE 6: (a) Myocardial apoptotic rate determined with TUNEL staining in control and doxorubicin-treated hearts. (b) and (c) Representative TUNEL staining (with DAPI costaining) in control and doxorubicin-treated hearts. Nuclei are stained blue. TUNEL positive nuclei are green/cyan. 10x, white bar $=200 \mu \mathrm{m},{ }^{*} P<0.05$ versus control. LV, left ventricle; RV, right ventricle; $n=4 /$ group.

Myocardial apoptosis and microvascular insufficiency both contribute to myocardial dysfunction in anthracyclineinduced HF [27-29, 36]. Indeed, doxorubicin-treated bovine hearts in our study exhibited both a 5 to 6 -fold increase in apoptotic rate and profound reductions in myocardial blood flow as quantified by regional microsphere distribution. As prior work has demonstrated that doxorubicin can induce apoptosis of cardiomyocytes and endothelial cells [25-27], these two phenomena may be interrelated. Specifically, endothelial cell death in the microvasculature may have contributed to the observed reductions in coronary blood flow. Indeed, we frequently observed foci of apoptotic nuclei in proximity to or within coronary arterioles in the heart, which suggests that at least some apoptotic cells were of vascular or endothelial origin.

As cardiomyopathic hearts generally show augmented interstitial fibrosis and collagen deposition [37], our finding of reduced interstitial collagen volume in doxorubicintreated hearts was surprising. However, prior work has demonstrated that doxorubicin upregulates and activates matrix metalloproteinases in the heart [38] and can also inhibit collagen synthesis [39] (both of which would favor collagen degradation). Indeed, in our study, the expression of profibrotic CTGF in the heart was not affected by doxorubicin, whereas MMP-2 (which promotes collagen breakdown) was upregulated, consistent with these prior observations. In a rat study of the cardiac matrix following a single injection of doxorubicin, a biphasic course of myocardial remodeling was observed. The initial response (up to 6 weeks after injection) was loss of the myocardial collagen matrix. At later time points, abnormal deposition of collagen produced focal myocardial scarring [40]. Hence, the interstitial remodeling after doxorubicin exposure may not be uniform and may depend on the stage of progression of doxorubicin-induced ventricular remodeling. It is possible that in our animals, longer periods of treatment or followup may have augmented collagen deposition, and greater fibrosis may have been observed. Moreover, as 


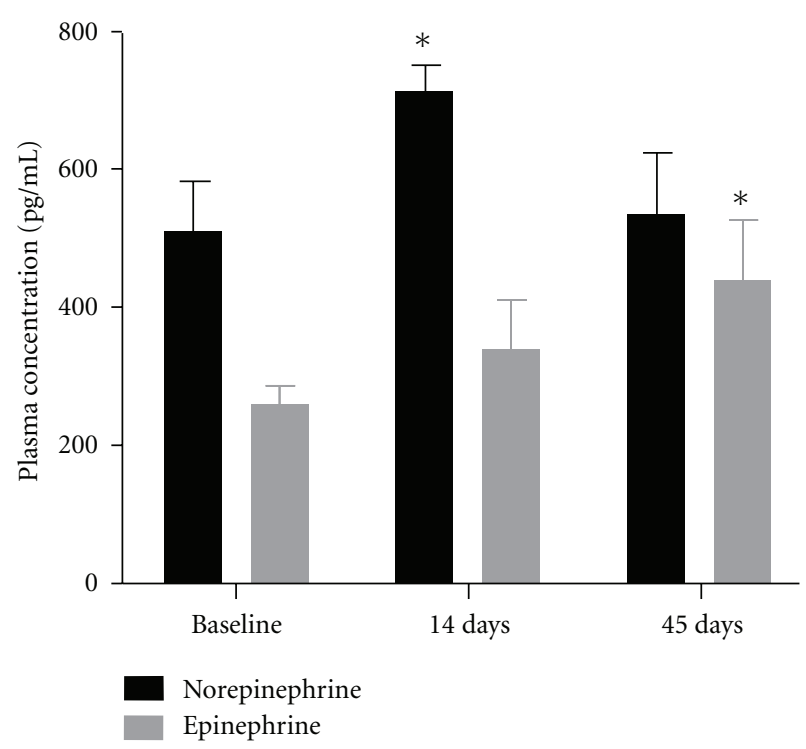

FIGURE 7: Plasma norepinephrine and epinephrine levels measured at the indicated time points during doxorubicin administration in calves. ${ }^{*} P<0.05$ versus baseline; $n=4$ /group.

myocardial fibrosis increases, ventricular chamber stiffness increases. The reduced collagen deposition that we observed may underlie the absence of diastolic filling pressure elevation in doxorubicin-treated hearts. The decrease in matrix protein may have increased chamber compliance and thereby maintained LVEDP at levels comparable to normal animals, despite the development of doxorubicin cardiomyopathy. The depressed peak $\mathrm{dP} / \mathrm{dt}$ and cardiac output in doxorubicin-treated animals despite equivalent LVEDP (preload) indicated significant contractile dysfunction in these animals. Filling pressure elevation and further hemodynamic compensation would have likely occurred over longer time periods that allowed for further progression of pathological remodeling.

4.1. Limitations. Our results must be interpreted in light of potential study limitations. In our study, the calves exhibited variability of response to doxorubicin toxicity. The one animal that was somewhat resistant to doxorubicin was a pure-breed Jersey calf, whereas the other three animals were mixed breed. Response variability has also been reported in other studies with doxorubicin. Astra et al. reported in a canine model of doxorubicin cardiomyopathy that one animal in their medium dose cohort showed no cardiac impairment, whereas all others animals showed severe impairment or died of heart failure [16]. Another study limitation was the small number of experimental animals, a situation that was mandated by unanticipated limitations in available resources for large animal maintenance. The small sample size increased the risk of type I statistical error (i.e., that the observed effects may have been driven by chance findings in just one animal) and type II statistical error (i.e., finding no difference between groups due to a small number of observations when a difference truly exists).
Nonetheless, despite the study of only four animals, the observed hemodynamic, structural, histological, biochemi$\mathrm{cal}$, and molecular changes were all sufficiently robust to establish the induction of LV dysfunction and pathological remodeling by doxorubicin. Moreover, the observed changes were consistent with multiple prior studies of doxorubicininduced cardiomyopathy in other animal models (described above), suggest that our results were experimentally valid and not simply the result of chance statistical variation.

These limitations notwithstanding, our results establish the validity and feasibility of a clinically relevant bovine model of doxorubicin-induced cardiomyopathy that shares many phenotypic similarities with human heart failure. This model may prove useful assess the pathophysiological responses to LVADs and associated adjunctive therapies in HF.

\section{Funding}

This work was supported in part by an American Heart Association Scientist Development Grant no. 0730319N (G.G.).

\section{Acknowledgments}

The authors acknowledge and thank Dr. Leslie Sherwood, Tim Horrell, Cary Woolard, Laura and Karen Lott, Ernest Cardwell, Justin Kingery, Robert Lewis, Menaka Nadar, and the University of Louisville Health Sciences veterinary staff for their assistance.

\section{References}

[1] J. A. Dixon and F. G. Spinale, "Large animal models of heart failure: a critical link in the translation of basic science to clinical practice," Circulation: Heart Failure, vol. 2, no. 3, pp. 262-271, 2009.

[2] W. M. Yarbrough and F. G. Spinale, "Large animal models of congestive heart failure: a critical step in translating basic observations into clinical applications," Journal of Nuclear Cardiology, vol. 10, no. 1, pp. 77-86, 2003.

[3] E. Bertho and G. A. Gagnon, "A comparative study in three dimension of the blood supply of the normal interventricular septum in human, canine, bovine, porcine, ovine and equine heart," Diseases of the Chest, vol. 46, pp. 251-262, 1964.

[4] D. Burkhoff, S. Klotz, and D. M. Mancini, "LVAD-induced reverse remodeling: basic and clinical implications for myocardial recovery," Journal of Cardiac Failure, vol. 12, no. 3, pp. 227-239, 2006.

[5] E. J. Birks, P. D. Tansley, J. Hardy et al., "Left ventricular assist device and drug therapy for the reversal of heart failure," New England Journal of Medicine, vol. 355, no. 18, pp. 1873-1884, 2006.

[6] M. A. Simon, R. L. Kormos, S. Murali et al., "Myocardial recovery using ventricular assist devices: prevalence, clinical characteristics, and outcomes," Circulation, vol. 112, no. 9, pp. I32-I36, 2005.

[7] A. H. Goldstein, G. Monreal, A. Kambara et al., "Partial support with a centrifugal left ventricular assist device reduces myocardial oxygen consumption in chronic, ischemic heart 
failure," Journal of Cardiac Failure, vol. 11, no. 2, pp. 142-151, 2005.

[8] A. Moritz, L. K. Fujimoto, G. Wollenek et al., "Sustained heart failure induced by repeated microsphere injections for left ventricular assist device testing," ASAIO Transactions, vol. 35, no. 3, pp. 455-458, 1989.

[9] K. Eya, E. Tuzun, J. Conger et al., "Effect of pump flow mode of novel left ventricular assist device upon end organ perfusion in dogs with doxorubicin induced heart failure," ASAIO Journal, vol. 51, no. 1, pp. 41-49, 2005.

[10] N. Borenstein, P. Bruneval, L. Behr et al., "An ovine model of chronic heart failure: echocardiographic and tissue doppler imaging characterization," Journal of Cardiac Surgery, vol. 21, no. 1, pp. 50-56, 2006.

[11] V.S. Chekanov, "A stable model of chronic bilateral ventricular insufficiency (dilated cardiomyopathy) induced by arteriovenous anastomosis and doxorubicin administration in sheep," Journal of Thoracic and Cardiovascular Surgery, vol. 117, no. 1, pp. 198-199, 1999.

[12] J. A. Magovern, I. Y. Christlieb, S. F. Badylak, G. C. Lantz, and R. L. Kao, "A model of left ventricular dysfunction caused by intracoronary adriamycin," Annals of Thoracic Surgery, vol. 53, no. 5, pp. 861-863, 1992.

[13] H. R. Shah, M. Vaynblat, G. Ramdev, J. N. Cunningham, and M. Chiavarelli, "Experimental cardiomyopathy as a model of chronic heart failure," Journal of Investigative Surgery, vol. 10, no. 6, pp. 387-396, 1997.

[14] C. W. Tomlinson, G. M. McGrath, and J. H. McNeill, "Adriamycin cardiomyopathy: pathological and membrane functional changes in a canine model with mild impairment of left ventricular function," Canadian Journal of Cardiology, vol. 2, no. 6, pp. 368-374, 1986.

[15] E. Monnet and E. C. Orton, "A canine model of heart failure by intracoronary Adriamycin injection: hemodynamic and energetic results," Journal of Cardiac Failure, vol. 5, no. 3, pp. 255-264, 1999.

[16] L. I. Astra, R. Hammond, K. Tarakji, and L. W. Stephenson, "Doxorubicin-induced canine CHF: advantages and disadvantages," Journal of Cardiac Surgery, vol. 18, no. 4, pp. 301-306, 2003.

[17] Y. Toyoda, M. Okada, and M. A. Kashem, "A canine model of dilated cardiomyopathy induced by repetitive intracoronary doxorubicin administration," Journal of Thoracic and Cardiovascular Surgery, vol. 115, no. 6, pp. 1367-1373, 1998.

[18] C. R. Bartoli, G. C. Wilson, G. A. Giridharan et al., "A novel subcutaneous counterpulsation device: acute hemodynamic efficacy during pharmacologically induced hypertension, hypotension, and heart failure," Artificial Organs, vol. 34, no. 7, pp. 537-545, 2010.

[19] G. Devlin, K. Matthews, G. McCracken et al., "An ovine model of chronic stable heart failure," Journal of Cardiac Failure, vol. 6, no. 2, pp. 140-143, 2000.

[20] C. R. Bartoli, K. Okabe, I. Akiyama, B. Coull, and J. J. Godleski, "Repeat microsphere delivery for serial measurement of regional blood perfusion in the chronically instrumented, conscious canine," Journal of Surgical Research, vol. 145, no. 1, pp. 135-141, 2008.

[21] S. L. Hale, K. J. Alker, and R. A. Kloner, "Evaluation of nonradioactive, colored microspheres for measurement of regional myocardial blood flow in dogs," Circulation, vol. 78, no. 2, pp. 428-434, 1988.

[22] S. C. Koenig, C. Woolard, G. Drew et al., "Integrated data acquisition system for medical device testing and physiology research in compliance with good laboratory practices,"
Biomedical Instrumentation and Technology, vol. 38, no. 3, pp. 229-240, 2004.

[23] T. Hamid, Y. Gu, R. V. Ortines et al., "Divergent tumor necrosis factor receptor-related remodeling responses in heart failure:Role of nuclear factor- $\kappa \mathrm{B}$ and inflammatory activation," Circulation, vol. 119, no. 10, pp. 1386-1397, 2009.

[24] G. Wang, T. Hamid, R. J. Keith et al., "Cardioprotective and antiapoptotic effects of heme oxygenase- 1 in the failing heart," Circulation, vol. 121, no. 17, pp. 1912-1925, 2010.

[25] T. Hamid, S. Z. Guo, J. R. Kingery, X. Xiang, B. Dawn, and S. D. Prabhu, "Cardiomyocyte NF- $\kappa \beta$ p 65 promotes adverse remodelling, apoptosis, and endoplasmic reticulum stress in heart failure," Cardiovascular Research, vol. 88, no. 3, 2010.

[26] M. Jessup and S. Brozena, "Heart failure," New England Journal of Medicine, vol. 348, no. 20, pp. 2007-2018, 2003.

[27] G. Takemura and H. Fujiwara, "Doxorubicin-induced cardiomyopathy from the cardiotoxic mechanisms to management," Progress in Cardiovascular Diseases, vol. 49, no. 5, pp. 330-352, 2007.

[28] K. L. Thompson, B. A. Rosenzweig, J. Zhang et al., "Early alterations in heart gene expression profiles associated with doxorubicin cardiotoxicity in rats," Cancer Chemotherapy and Pharmacology, pp. 1-12, 2009.

[29] S. Wu, YU. S. Ko, M. S. Teng et al., "Adriamycin-induced cardiomyocyte and endothelial cell apoptosis: in vitro and in vivo studies," Journal of Molecular and Cellular Cardiology, vol. 34, no. 12, pp. 1595-1607, 2002.

[30] J. H. Doroshow, G. Y. Locker, and C. E. Myers, "Enzymatic defenses of the mouse heart against reactive oxygen metabolites. Alterations produced by doxorubicin," Journal of Clinical Investigation, vol. 65, no. 1, pp. 128-135, 1980.

[31] E. Monnet and J. C. Chachques, "Animal models of heart failure: what is new?" Annals of Thoracic Surgery, vol. 79, no. 4, pp. 1445-1453, 2005.

[32] I. D. Gregoric, D. Tamez, M. N. Karabulut et al., "Aortic valve implantation in the calf: a successful approach using

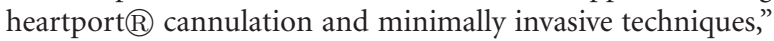
Journal of Heart Valve Disease, vol. 10, no. 5, pp. 675-680, 2001.

[33] H. K. Chee, E. Tuzun, M. Ferrari et al., "Baseline hemodynamic and echocardiographic indices in anesthetized calves," ASAIO Journal, vol. 50, no. 3, pp. 267-271, 2004.

[34] H. C. Lin, J. C. Thurmon, W. J. Tranquilli, G. J. Benson, and W. A. Olson, "Hemodynamic response of calves to tiletamine-zolazepam-xylazine anesthesia," American Journal of Veterinary Research, vol. 52, no. 10, pp. 1606-1610, 1991.

[35] G. D. Hallowell, T. J. Potter, and I. M. Bowen, "Methods and normal values for echocardiography in adult dairy cattle," Journal of Veterinary Cardiology, vol. 9, no. 2, pp. 91-98, 2007.

[36] R. B. Wanless, I. S. Anand, J. Gurden, P. Harris, and P. A. Poole-Wilson, "Regional blood flow and hemodynamics in the rabbit with adriamycin cardiomyopathy: effects of isosorbide dinitrate, dobutamine and captopril," Journal of Pharmacology and Experimental Therapeutics, vol. 243, no. 3, pp. 1101-1106, 1987.

[37] S. D. Prabhu, "Post-infarction ventricular remodeling: an array of molecular events," Journal of Molecular and Cellular Cardiology, vol. 38, no. 4, pp. 547-550, 2005.

[38] A. Goetzenich, N. Hatam, A. Zernecke et al., "Alteration of matrix metalloproteinases in selective left ventricular adriamycin-induced cardiomyopathy in the pig," Journal of Heart and Lung Transplantation, vol. 28, no. 10, pp. 10871093, 2009. 
[39] A. Muszyńska, S. Wo, and J. Pa, "The mechanism for anthracycline-induced inhibition of collagen biosynthesis," European Journal of Pharmacology, vol. 411, no. 1-2, pp. 1725, 2001.

[40] J. B. Caulfield and V. Bittner, "Cardiac matrix alterations induced by adriamycin," American Journal of Pathology, vol. 133, no. 2, pp. 298-305, 1988. 


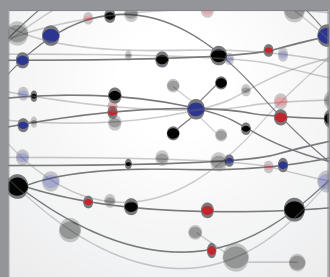

The Scientific World Journal
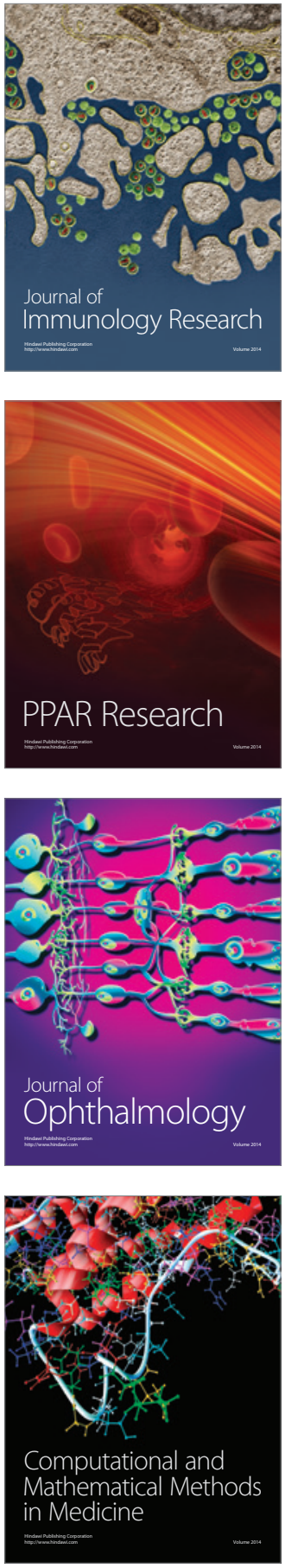

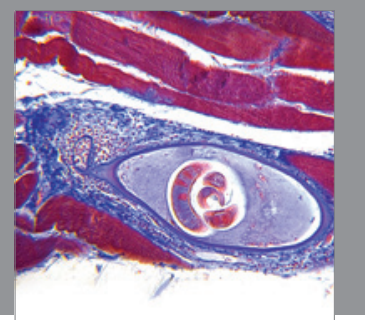

Gastroenterology

Research and Practice
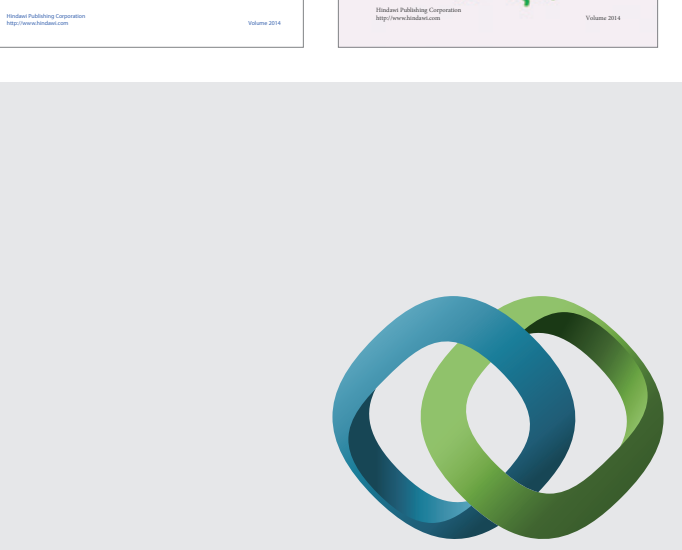

\section{Hindawi}

Submit your manuscripts at

http://www.hindawi.com
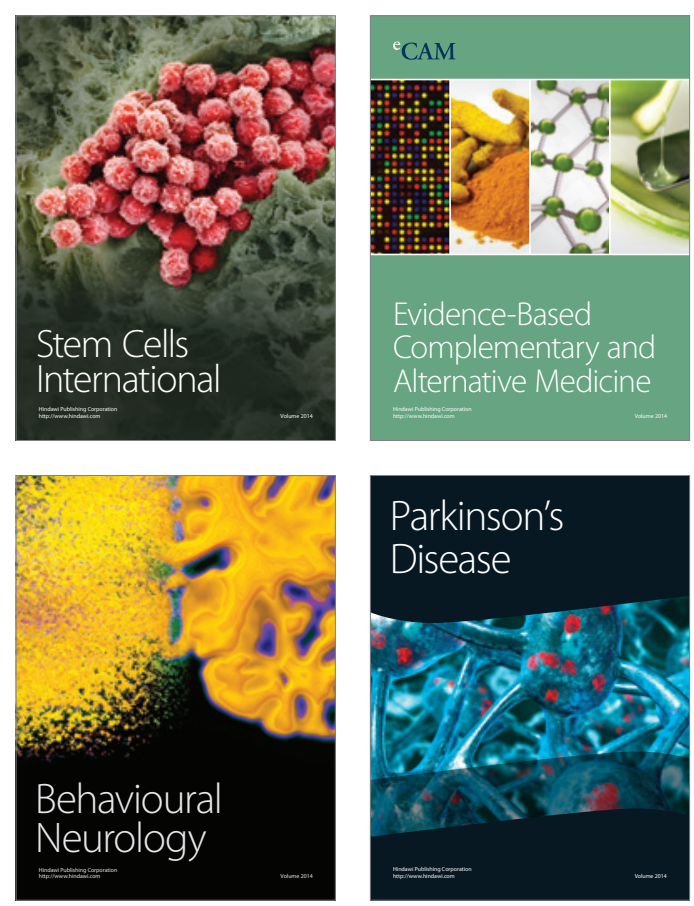

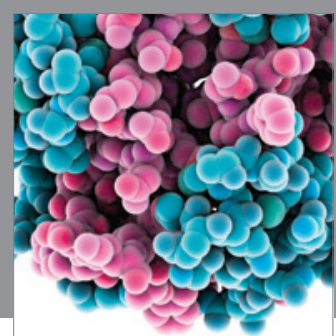

Journal of
Diabetes Research

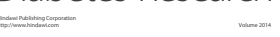

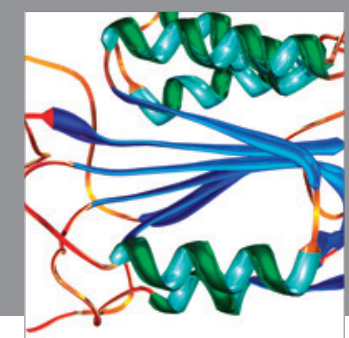

Disease Markers
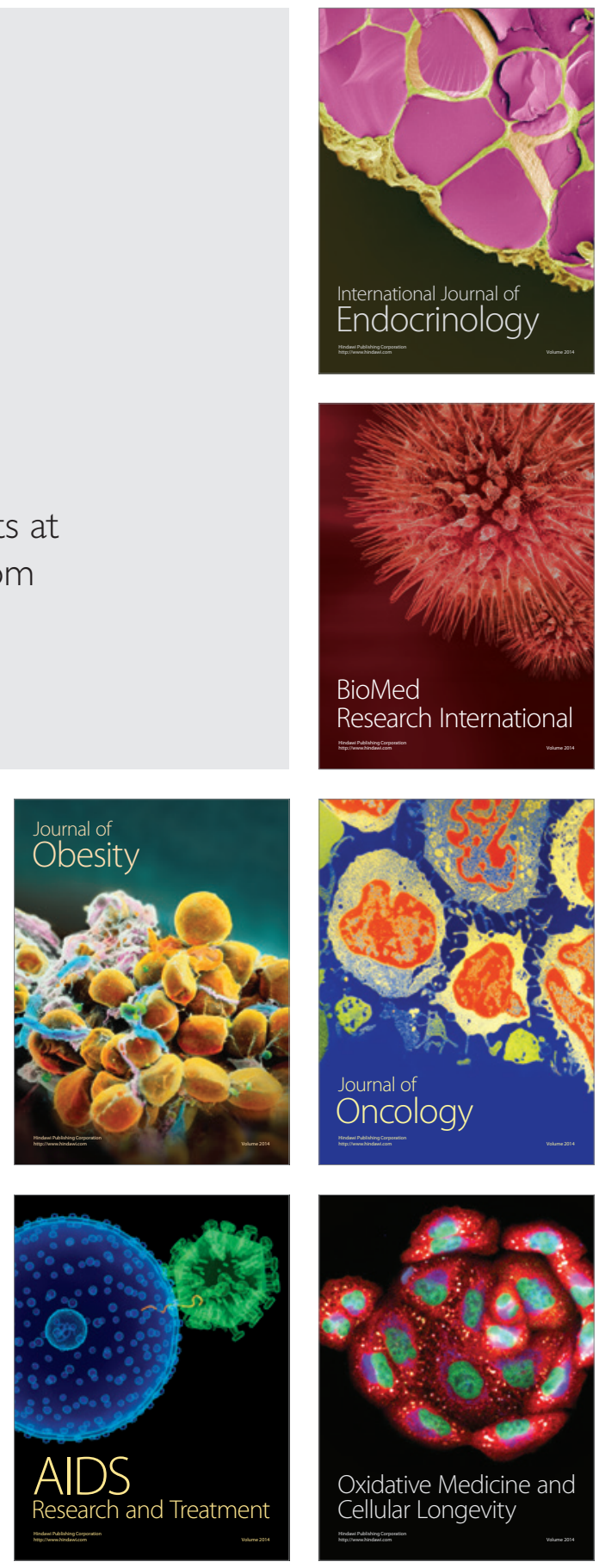